Olivet Nazarene University

Digital Commons @ Olivet

\title{
Protective Effects of the Novel Phytonutrient Spectra7 Against Intestinal Tight Junction Disruption: Composition Matters
}

Erin Olson

erinelsaolson@gmail.com

Follow this and additional works at: https://digitalcommons.olivet.edu/honr_proj

Part of the Biology Commons, and the Cellular and Molecular Physiology Commons

\section{Recommended Citation}

Olson, Erin, "Protective Effects of the Novel Phytonutrient Spectra7 Against Intestinal Tight Junction Disruption: Composition Matters" (2020). Honors Program Projects. 114.

https://digitalcommons.olivet.edu/honr_proj/114

This Article is brought to you for free and open access by the Honors Program at Digital Commons @ Olivet. It has been accepted for inclusion in Honors Program Projects by an authorized administrator of Digital Commons @ Olivet. For more information, please contact digitalcommons@olivet.edu. 


\title{
Protective Effects of the Novel Phytonutrient S7 Against Intestinal Tight Junction Disruption: Composition Matters
}

\author{
Erin E. Olson
}

\begin{abstract}
AKNOWLEDGEMENTS
I would like to express my most sincere gratitude to my research mentor, Dr. Daniel Sharda. His expertise, guidance, and support throughout the process of project design and execution were truly invaluable. I am thankful for his constant encouragement as we navigated hurdles and interpreted endlessly interesting data, as well as for his constructive feedback on the art of scientific writing. I would also like to thank Alexi Zastrow, who worked with Dr. Sharda and me during summer 2019 to conduct a parallel study, investigating the impact of S7 on M1-macrophage activation. Her positivity and constant encouragement as well as assistance with reagent preparation and cell passaging were greatly appreciated. Thanks also to my Honors Cohort professors, Dr. Stephen Case, Dr. Beth Schurman, Dr. Brian Stipp, and Professor Eric Young, who fostered a love of scholarship and critical analysis and provided support and feedback during early stages of project development. Special thanks also to Carrie Yuan at Hunter Laboratory, who instructed Dr. Sharda and me in the use of the TEER apparatus and provided valuable troubleshooting advice.

Financial support for this research was provided by the ONU Honors Department, the Elbert Pence and Fanny Boyce Undergraduate Summer Research Experience, and the Hippenhammer Faculty Research Grant. Caco-2 cells were a gift from Hunter Laboratory at Northwestern University. S7 reagents and Restore were gifts from Futureceuticals and Biomic Sciences respectively.
\end{abstract}




\begin{abstract}
The prevalence of intestinal inflammatory diseases is increasing, and pharmacologic agents for intervention are currently limited. Preserving epithelial tight junction (TJ) integrity and preventing underlying immune cell activation by intestinal bacteria are key targets for abrogating the perpetual inflammatory cycle that plagues these diseases. Phytonutrients have shown promise for their ability to reduce cellular inflammation, but the extent of their efficacy in an intestinal model of inflammation is not well understood. Here, we hypothesized that S7, a novel phytonutrient derived from extracts rich in curcuminoids and catechins, would reduce immune cell inflammation and preserve TJ integrity in an in vitro co-culture model of intestinal inflammation. We further investigated whether a curcumin-containing formulation (S7-C) or its metabolite, tetrahydrocurcumin (S7-THC) would similarly preserve TJ integrity.

An in vitro intestinal co-culture model was established by seeding Caco-2 epithelial cells on semipermeable transwell inserts 21 days prior to the addition of RAW264.7 macrophages in the basolateral chamber. Macrophages were next stimulated with $10 \mathrm{ng} / \mathrm{ml}$ lipopolysaccharide (LPS) to induce inflammation, and subsequent TJ disruption in the co-cultured Caco-2 cells was assessed by transepithelial electrical resistance (TEER) using epithelial ohmmeter chopstick electrodes. We found that administration of S7-THC containing 1-5 $\mu \mathrm{M}$ THC produced dose dependent mitigation of LPS-induced decreases in TEER and approached the efficacy of the pharmacologic agent, budesonide. However, S7-C at $5 \mu \mathrm{M}$ curcumin was unable to preserve TEER, suggesting that the specific combination of phytonutrients is important for preventing inflammation-induced TJ disruption. We also found that, though apical application (Caco-2 only) of budesonide was sufficient for preserving TEER in our model, S7-THC required both apical (Caco-2) and basolateral (RAW264.7) treatment, suggesting that reducing macrophage inflammation is important for limiting epithelial TJ disruption in this context. Interestingly, S7-C was more effective than S7-THC or budesonide at reducing inflammatory basolateral nitric oxide (NO) production as determined by the Griess assay. This suggests that, though S7-C more effectively reduces this aspect of inflammation, another inflammatory mediator is responsible for conveying TJ disruption and is governed differentially by S7-THC. Further support of this comes from our finding that S7-THC, but not S7-C, ameliorated the LPS-induced increase in myosin light chain kinase (MLCK) expression in Caco-2 cells as determined by Western blot. Together, these findings suggest that phytonutrients such as S7-THC have prophylactic potential in the preservation of TJ integrity, and the specific composition of these phytonutrients matters.
\end{abstract}

Keywords: Intestinal tight junctions, intestinal inflammation, intestinal barrier, lipopolysaccharide, Caco-2 cells, transepithelial electrical resistance, phytonutrients, S7 